\title{
Systemic Complement Activation, Lung Injury, and Products of Lipid Peroxidation
}

Peter A. Ward, Gerd O. Till, John R. Hatherill, Thomas M. Annesley, and Robin G. Kunkel

Department of Pathology, The University of Michigan Medical School, Ann Arbor, Michigan 48109-0010

\section{Abstract}

Previously we have demonstrated that systemic activation of the complement system after intravenous injection of cobra venom factor (CVF) results in acute lung injury as reflected by increases in the vascular permeability of the lung as well as by morphologic evidence of damage to lung vascular endothelial cells. In using the vascular permeability of the lung as the reference, the current studies show a quantitative correlation between lung injury and the appearance in plasma of lipid peroxidation products (conjugated dienes) as well as increased concentrations of lactic dehydrogenase (LDH) and one of its isoenzymes (LDH-4). After injection of CVF, extracts of lungs also showed elevated levels of conjugated dienes, whereas no elevations were found in extracts of liver, kidney, and spleen. There was no evidence in CVF-injected rats of renal or hepatic injury as reflected by the lack of development of proteinuria and the failure to detect increased serum levels of liver-related enzymes. Other peroxidation products identified in plasma of CVF-injected rats involved hydroperoxides and fluorescent compounds with features of Schiff bases. Not surprisingly, malondialdehyde was not found to be a reliable plasma indicator of lipid peroxidation associated with oxygen radical-mediated lung vascular injury. In using a model of oxygen radicalindependent lung injury induced by oleic acid, although large amounts of LDH and LDH-4 were found in the plasma, no increases in plasma levels of conjugated dienes were detected. In CVF-injected animals treated with interventions protective against lung injury (neutrophil depletion, catalase, hydroxyl radical scavengers, or iron chelators), there were striking reductions in the plasma levels of conjugated dienes, hydroperoxides, and fluorochromic products. Morphometric analysis of lung sections revealed that the protective interventions did not interfere with the accumulation of neutrophils in lung interstitial capillaries after systemic activation of complement. In vitro studies with phorbol-stimulated neutrophils failed to demonstrate appearance of conjugated dienes, suggesting that the dienes appearing in plasma of CVF-injected animals are not the result of autotoxic changes in neutrophils. The data presented in this paper suggest that acute lung injury mediated by oxygen radicals derived from phagocytic cells can be monitored by the appearance in plasma of products of lipid peroxidation.

Address reprint requests to Dr. Ward, Box 045, M5240 Medical Science I, 1315 Catherine Road, Ann Arbor, MI 48109-0010.

Received for publication 14 October 1984 and in revised form 14 March 1985.

J. Clin. Invest.

(c) The American Society for Clinical Investigation, Inc. 0021-9738/85/08/0517/11 $\$ 1.00$

Volume 76, August 1985, 517-527

\section{Introduction}

Systemic activation of the complement system in rats leads to acute lung injury in which interstitial vascular endothelial cells are the chief target of injury. The pathophysiologic processes involved include the activation of blood neutrophils and their generation of toxic oxygen products which are directly responsible for injury of lung vascular endothelial cells (1). This model of acute lung injury is dependent on the generation of $\mathrm{H}_{2} \mathrm{O}_{2}$ and the presence of ionic iron, as determined by the protective effects of catalase and iron chelators (2). Based on this information as well as other evidence, it has been concluded that the injury of lung vascular endothelial cells after intravascular activation of complement is due to the generation of the highly reactive and short-lived hydroxyl radical (HO'), which may be generated by the following sequence:

$$
\begin{aligned}
& \mathrm{Fe}^{3+}+\mathrm{O}_{2}^{-} \rightarrow \mathrm{Fe}^{2+}+\mathrm{O}_{2} \\
& \mathrm{Fe}^{2+}+\mathrm{H}_{2} \mathrm{O}_{2} \rightarrow \mathrm{Fe}^{3+}+\mathrm{HO}^{-}+\mathrm{OH}^{-}
\end{aligned}
$$

In this Fenton reaction, iron acts as a redox agent for the transfer of electrons from and to oxygen products to form ultimately the highly toxic $\mathrm{H}_{2} \mathrm{O}_{2}$ derivative, $\mathrm{HO}^{\circ}$.

The manner in which toxic oxygen products bring about injury of tissues is not well understood. It is well known that polyunsaturated fatty acids undergo lipid peroxidation after exposure to dioxygen or its derivatives (3). There is also abundant evidence that iron facilitates lipid peroxidation in vitro although there is considerable debate as to whether or not iron is directly involved through the formation of the postulated perferryl ion or whether iron functions to bring about lipid peroxidation by facilitating the generation of $\mathrm{HO}^{\circ}$ (reviewed in reference 4). Products of lipid peroxidation include hydroperoxides, conjugated dienes, malonaldehyde/malondialdehyde, and fluorochromic proteins or amino-containing phospholipids that develop intra- or intermolecular cross-links with malonaldehyde or malondialdehyde and demonstrate features of conjugated Schiff bases (reviewed in references 5 and 6). The studies to be described were designed to determine whether or not lipid peroxidation products could be demonstrated in vivo after systemic complement activation and the extent to which the appearance of these products is affected by interventions that protect against complement-induced acute lung injury. The studies demonstrate that lipid peroxidation products appear in plasma and in lung tissue in the course of acute lung injury after systemic activation of complement, that there is a direct quantitative relationship between the intensity of acute lung injury and the levels of products of lipid peroxidation, and that interventions protective against lung injury correspondingly alter in a quantitative relationship the appearance of products of lipid peroxidation. 


\section{Methods}

Animal models. Young adult male Long-Evans pathogen-free rats weighing $300-350 \mathrm{~g}$ were used. They were intravenously injected with $2.5 \mathrm{U} / 100 \mathrm{~g}$ of body weight (in $0.5 \mathrm{ml}$ of sterile saline) of cobra venom factor (CVF) ${ }^{1}$ to produce complement-mediated acute lung injury (1). As previously described, lung injury was determined by the extravascular leakage of ${ }^{125} \mathrm{I}$-bovine serum albumin and recorded as the ratio of radioactivity in lungs that had been vascularly perfused with saline to that amount of radioactivity in $1.0 \mathrm{ml}$ of blood obtained at the time of sacrifice. As described below, appearance of lactate dehydrogenase (LDH) in plasma was also used as an indicator of lung injury.

A second animal model of lung injury in rats produced by the intravenous injection of oleic acid was employed (7). This model was used because it was demonstrated recently that oxygen radicals are probably not involved in the pulmonary vascular endothelial cell injury inasmuch as prior treatment of animals with superoxide dismutase (SOD), catalase, or dimethyl sulfoxide (DMSO) fails to protect against the lung injury (8). For these experiments $50 \mu \mathrm{g}$ of oleic acid (purchased from Sigma Chemical Co., St. Louis, MO) dissolved in $200 \mu$ l of saline containing $0.1 \%$ bovine serum albumin was used. Animals were sacrificed at $1 \mathrm{~h}$, the peak of lung injury $(7,8)$, the change in vascular permeability was measured as described above, and the plasma was obtained for measurements of conjugated dienes as described below.

Plasma samples. Plasma was obtained from rats by heart puncture or by aspiration of blood from the posterior vena cava through using heparinized syringes such that the final concentration of heparin was $5 \mathrm{U} / \mathrm{ml}$ of blood. The blood was then immediately subjected to centrifugation, and the resulting plasma was kept at $4^{\circ} \mathrm{C}$ until appropriate assays were carried out. If retained, plasma was stored at $-70^{\circ} \mathrm{C}$ for analysis 1 wk later. (We have determined that $<5 \%$ of $\mathrm{LDH}$ activity is altered by freezing.) Except for the LDH analyses, all measurements were made within $2 \mathrm{~h}$ after obtaining the plasma samples.

$L D H$ isoenzymes. Total LDH was measured on a COBAS-Bio centrifugal analyzer (Roche Analytical Instruments, Inc., Nutley, NJ) by using the procedure proposed by Gay et al. (9). LDH isoenzyme separation was performed by using agarose gel electrophoresis (Beckman Paragon System, Beckman Instruments, Inc., Palo Alto, CA). When tissue LDH content was studied, tissues were homogenized in phosphatebuffered saline (see below), and the soluble extracts obtained by centrifugation were measured for total LDH content. These samples were diluted as necessary to an LDH activity of $700 \mathrm{U} /$ liter. $5 \mu \mathrm{l}$ of specimen were then applied to the gel, followed by electrophoresis for $20 \mathrm{~min}$ at $100 \mathrm{~V}$. Isoenzymes were detected by linking the reaction described by Gay et al. (9) to the reduction of nitroblue tetrazolium chloride, yielding a stable blue band for each isoenzyme of LDH. The percentage contribution of isoenzymes was determined by scanning densitometry that used a Beckman Instruments, Inc. model CDS-200 densitometer. Multiplication of the percentage of a given LDH activity (established by densitometry) by total activity yielded a numerical estimation of a given LDH isoenzyme activity in serum. The isoenzyme pattern in a serum specimen was then correlated with organ isoenzyme patterns obtained from various tissues.

Liver-related enzymes. Sera were collected $30 \mathrm{~min}$ after intravenous injection of CVF, as described above, and enzyme assays were carried out within a few hours on a COBAS centrifugal analyzer. Aspartate aminotransferase, alanine aminotransferase, and leucine aminopeptidase were measured using the standard coupled enzyme assay (10-12) whereas alkaline phosphatase was analyzed using $p$-nitrophenyl phosphate as substrate (13).

Interventional therapy. Several different agents known to have

1. Abbreviations used in this paper: CVF, cobra venom factor; DMSO, dimethyl sulfoxide; DMTU, dimethyl thiourea; LDH, lactate dehydrogenase; PEG, polyethylene glycol; PMA, phorbol myristate acetate; SOD, superoxide dismutase. protective effects against acute lung injury after intravenous injection of CVF $(1,2)$ were studied, and these results were correlated with the effects of these interventions on the appearances of products of lipid peroxidation. Unless otherwise indicated, all agents were given intravenously immediately before injection of CVF. The hydroxyl radical scavengers DMSO $(1.5 \mathrm{ml} / \mathrm{kg})$ (Fisher Scientific Co., Fair Lawn, NJ) and dimethyl thiourea (DMTU) $(1,000 \mathrm{mg} / \mathrm{kg}$ ) (Alfa Products, Danvers, MA) were given intraperitoneally and intravenously, respectively. The iron chelator deferoxamine mesylate (Ciba-Geigy Corp., Summit, NJ) was injected intravenously in the amount of $15 \mathrm{mg} / \mathrm{kg}$ body weight. Iron-saturated lactoferrin and its non-iron-containing form (apolactoferrin) purified from human breast milk were each employed in a dose of $10 \mathrm{mg}$. The preparation of these reagents is described in a recent publication (2). Iron-saturated deferoxamine ( $290 \%$ saturation) was prepared by dissolving appropriate amounts of $\mathrm{FeCl}_{3}$ in an aqueous solution of deferoxamine. Polyethylene-glycol-derivatized catalase (PEGcatalase) and PEG-superoxide dismutase (PEG-SOD) (Enzon Inc., Piscataway, $\mathrm{NJ})$ were injected intravenously $(1,200 \mathrm{U} / \mathrm{kg}$ body weight) $10 \mathrm{~min}$ before the injection of CVF. As a control, a sample of PEGcatalase was first heat-inactivated at $100^{\circ} \mathrm{C}$ for $10 \mathrm{~min}$.

Neutrophil depletion. Depletion of circulating neutrophils $(<500 /$ $\mathrm{mm}^{3}$ blood) was achieved by the intraperitoneal injection of a rabbit antiserum against rat neutrophils $(0.5 \mathrm{ml} / 100 \mathrm{~g}$ of body weight) $18 \mathrm{~h}$ before injection of CVF, as described previously (1).

Lipid peroxidation products. Quantitative estimation of products of lipid peroxidation included assays for conjugated dienes, malondialdehyde, hydroperoxides, and fluorochromic products with features of Schiff bases. The procedural details can be found elsewhere (14). Conjugated dienes extracted from plasma or tissues using a 2:1 (vol/ vol) mixture of chloroform and methanol. For plasma, $7 \mathrm{ml}$ of the chloroform-methanol mixture, preheated to $45^{\circ} \mathrm{C}$, were added to 0.5 $\mathrm{ml}$ of plasma. The mixture was then vigorously mixed (with a vortex machine) for $2 \mathrm{~min}$, then subjected to centrifugation $(1,500 \mathrm{~g})$ for 5 min. $5 \mathrm{ml}$ from the lower layer (chloroform) was aspirated and mixed with $2.0 \mathrm{ml}$ of distilled water acidified with $0.1 \mathrm{~N} \mathrm{HCl}$ to a pH of 2.5 . After agitation that used a vortex instrument, the material was again subjected to centrifugation (as described above), and $2.0 \mathrm{ml}$ of the lower layer was aspirated, transferred to a test tube $(12 \times 75 \mathrm{~mm})$, and dried under a flow of nitrogen gas. The residue was reconstituted with $0.5 \mathrm{ml}$ of heptane and measured spectrophotometrically at 233 $\mathrm{nm}$. Tissues were extracted in a similar fashion, with $0.8 \mathrm{ml}$ of distilled water being added for each $1.0 \mathrm{~g}$ of wet tissue. The material was then homogenized in a Polytron instrument (VWR Scientific, Inc., Detroit, MI) with the speed indicator set at 7 for $1.0 \mathrm{~min}$. Subsequently, for 1 $\mathrm{g}$ of tissue wet weight, $6.0 \mathrm{ml}$ of chloroform-methanol $(1: 2, \mathrm{vol} / \mathrm{vol})$ were added, followed by agitation for $2 \mathrm{~min}$ with a vortex instrument. Then, $2.0 \mathrm{ml}$ of this mixture (per $1.0 \mathrm{~g}$ of wet tissue) were added, followed by use of the vortex instrument for $30 \mathrm{~s}$. $2 \mathrm{ml}$ of acidified water (as above) were subsequently added (per $1.0 \mathrm{~g}$ of wet tissue), followed by mixing with a vortex instrument for another $30 \mathrm{~s}$. Finally, the material was cleared by centrifugation, and the chloroform layer was aspirated and treated as described above. Malondialdehyde was measured as thiobarbituric acid activity in the following manner. $1 \mathrm{ml}$ of sample was added to a 1:1:1 (vol/vol/vol) solution of trichloroacetic acid ( $15 \%$, wt/vol), thiobarbituric acid $(0.375 \%$, wt/vol), and hydrochloric acid, $0.25 \mathrm{~N}$. The mixture was heated at $100^{\circ} \mathrm{C}$ for $25 \mathrm{~min}$. Then the supernatant was obtained by centrifugation $(1,500 \mathrm{~g}$ for $5 \mathrm{~min})$, and the absorbance at $535 \mathrm{~nm}$ was determined. Immediately before the addition of the sample, butylated hydroxytoluene was added in a final concentration of $0.01 \%(\mathrm{wt} / \mathrm{vol})$ in order to block further lipid peroxidation. Lipid hydroperoxides were measured according to the iodometric assay (14). Briefly, $1.0 \mathrm{ml}$ of sample was mixed with $7.0 \mathrm{ml}$ of chloroform-methanol (2:1, vol/vol), agitated with a vortex instrument for $2 \mathrm{~min}$, and then subjected to centrifugation $(1,500 \mathrm{~g})$ for $5 \mathrm{~min}$. Aspiration of $5.0 \mathrm{ml}$ of the lower chloroform layer was carried out with the chloroform extract then being dried under nitrogen. When dry, $1.0 \mathrm{ml}$ of an acetic acid-chloroform mixture $(3: 2$, vol/vol) was 
added, followed by $0.05 \mathrm{ml}$ of potassium iodide $(1.2 \mathrm{~g} / \mathrm{vol})$ and rapid shaking of the sample which was then shielded from light for $5 \mathrm{~min}$, followed by addition of $3.0 \mathrm{ml}$ of cadmium acetate $(0.5 \mathrm{~g} \%)$. The solution was shaken vigorously, cleared by centrifugation, and the upper (water) phase was aspirated and the absorbance at $353 \mathrm{~nm}$ was determined. A standard curve of absorbance for the assay for hydroperoxides was developed for reference purposes using cumene hydroperoxide (Sigma Chemical Co.). Fluorescent products were isolated by extracting $0.5-\mathrm{ml}$ aliquots of plasma with $7.0 \mathrm{ml}$ of chloroformmethanol (2:1, vol/vol) followed by the addition of $2 \mathrm{ml}$ of acidic $\mathrm{H}_{2} \mathrm{O}$ (as described above). The methanol- $\mathrm{H}_{2} \mathrm{O}$ fractions were analyzed by fluorescence at $430 \mathrm{~nm}$ when excited at $360 \mathrm{~nm}$ with an SF-330Varian spectrofluorometer (Varian Associates, Inc., Palo Alto, CA) previously standardized with $1 \mu \mathrm{g} / \mathrm{ml}$ quinine sulfate.

Morphometric analysis. To determine whether or not interventions protective against CVF-induced lung injury alter the sequestration of neutrophils within lung interstitial capillaries, for each protective intervention employed two different animals were studied under the conditions described above. At the time of sacrifice $(30 \mathrm{~min}$ after injection of CVF), the lungs were fixed by airway inflation that used 4\% glutaraldehyde containing $0.1 \mathrm{M}$ cacodylate buffer ( $\mathrm{pH}$ 7.4). Lungs were then sliced and the sections were dehydrated with increasing amounts of alcohol and infiltrated and embedded in epon. Then, 1$\mu \mathrm{m}$-thick sections were obtained and stained with toluidine blue. The sections were mounted on glass slides and examined under light microscopy $(\times 40)$. For microscopic examination, eight randomly obtained sections of lung (from two animals) were employed. In each tissue section, five different microscopic fields were examined (for a total of $\mathbf{4 0}$ fields for each intervention employed). All interstitial capillaries observed within each microscopic field were examined and the numbers of neutrophils within each capillary determined. Results were expressed as mean \pm standard error of the mean neutrophils per 40 high power $(\times 40)$ fields. The data were analyzed by calculating $P$ values using Student's $t$ test.

Conjugated diene formation in stimulated neutrophils. Neutrophils were obtained from glycogen-induced peritoneal exudates, washed in phosphate-buffered ( $\mathrm{pH}$ 7.4) Hanks' balanced salt solution, and resuspended in the same solution. Neutrophils $\left(2 \times 10^{6}\right)$ in $1.0 \mathrm{ml}$ were stimulated with $200 \mathrm{ng}$ of phorbol myristate acetate (PMA) for varying periods of time (up to $1 \mathrm{~h}$ ) at $37^{\circ} \mathrm{C}$. Under these conditions, maximal generation of $\mathrm{O}_{2}^{-}$and $\mathrm{H}_{2} \mathrm{O}_{2}$ occurs (15). Incubations were terminated by the addition of $7.0 \mathrm{ml}$ of chloroform/methanol $(2: 1, \mathrm{vol} / \mathrm{vol})$ to each $1.0 \mathrm{ml}$ of cell suspension. Extraction of conjugated dienes was carried out and analyzed, as described above.

Statistical analysis. Data were expressed as mean \pm standard error of the mean. For determination of significance of the data, the $t$ test was employed.

\section{Results}

Profiles of LDH isoenzymes in tissues. To assess whether LDH isoenzyme patterns might be a useful indicator of lung injury, extracts from several different rat tissues, including erythrocytes, were obtained and the LDH isoenzyme profiles were determined as assessed by electrophoretic separation and densitometric analysis. As is demonstrated in Fig. 1 and Table I, plasma or serum and all tissues except heart and brain had prominent LDH isoenzyme activity in position 5 . As determined by densitometric analysis of the samples shown in Fig. 1, the ratio of percent LDH-4 and LDH-5 in lung was 12:86, with faint traces ( $<2 \%$ of total $\mathrm{LDH}$ ) for each of the other isoenzymes (Table I). In the case of extracts from heart, the predominant LDH isoenzymes were in positions 1, 2, and 3; kidney and brain had significant amounts $(>5 \%)$ of each of the five isoenzymes although the ratios were somewhat different for

\section{LDH ISOENZYMES}

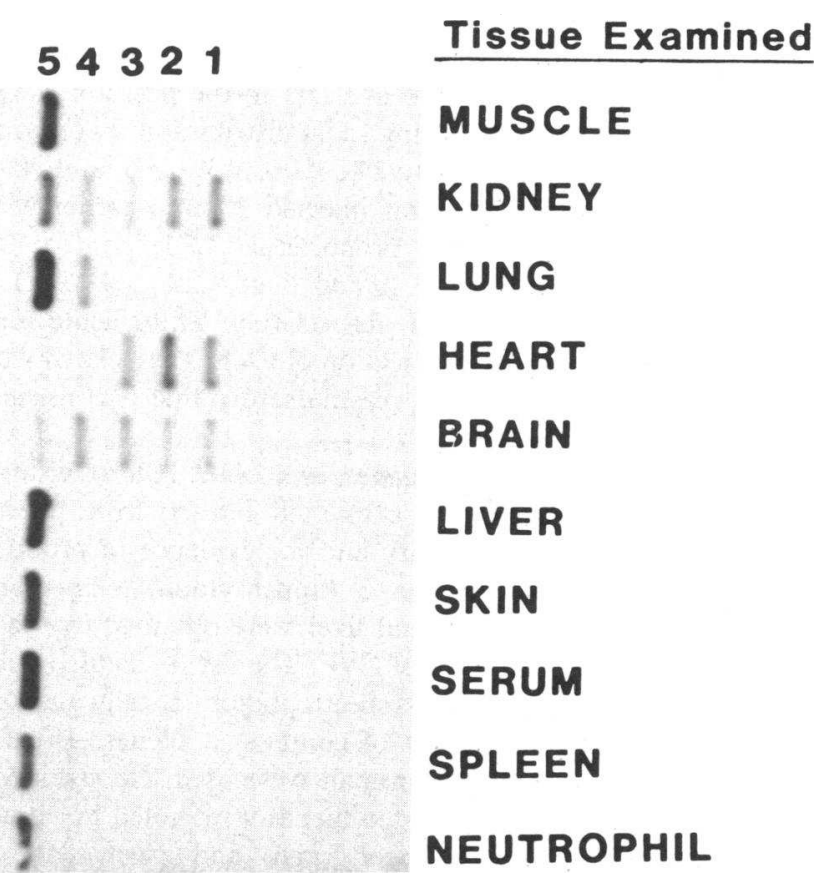

Figure 1. LDH isoenzyme profiles of extracts from a variety of normal rat tissues. Point of application was on the left (near position 3 ); anode was to the right.

the two organs. Muscle, liver, skin, normal rat serum, erythrocytes, and rat peritoneal neutrophils had only isoenzymes in position 5 whereas the spleen extract had a similar pattern but with a small amount $(2.5 \%)$ of isoenzyme in position 4 . Thus, the lung appeared to have a fairly characteristic LDH isoenzyme profile.

Based on this type of analysis, it appeared reasonable to employ this methodology for monitoring plasma of animals for evidence of lung damage. The assumption that the presence of LDH-4 is indicative of lung origin is only valid if the ratios of LDH-4 and 5 are similar to the lung profile of $\mathrm{LDH}$ isoenzyme described in Table I and if there are, at most, only

Table I. Tissue and Organ Profiles of LDH Isoenzyme Activity

\begin{tabular}{|c|c|c|c|c|c|}
\hline \multirow[b]{2}{*}{ Tissue } & \multicolumn{5}{|c|}{ LDH isoenzyme position } \\
\hline & 1 & 2 & 3 & 4 & 5 \\
\hline & \multicolumn{5}{|c|}{ Percent of total } \\
\hline Lung & $<1$ & $<1$ & $<1$ & 12 & 86 \\
\hline Heart & 31 & 43 & 20 & 4 & 2 \\
\hline Kidney & 23.5 & 19 & 6 & 12 & 39.5 \\
\hline Brain & 17 & 12 & 24 & 32 & 15 \\
\hline Muscle & $<1$ & $<1$ & $<1$ & $<1$ & $>99$ \\
\hline Liver & $<1$ & $<1$ & $<1$ & $<1$ & $>99$ \\
\hline Skin & $<1$ & $<1$ & $<1$ & 1 & 99 \\
\hline Spleen & $<1$ & $<1$ & $<1$ & 2.5 & 97.5 \\
\hline Serum & $<1$ & $<1$ & $<1$ & $<1$ & $>99$ \\
\hline Neutrophils & $<1$ & $<1$ & $<1$ & $<1$ & $>99$ \\
\hline Erythrocytes & $<1$ & $<1$ & $<1$ & $<1$ & $>99$ \\
\hline
\end{tabular}


traces of LDH-1, 2, and 3 (not exceeding 5\% in aggregate of the total LDH isoenzyme profile). In animals injected with CVF, there was a striking change in the plasma profile of LDH isoenzyme, as shown in Fig. $2.30 \mathrm{~min}$ after injection of CVF, a prominent appearance of LDH in the position 4 was demonstrated, with the following LDH distribution: isoenzyme positions $1,2,3,4$, and 5 were $2 \%,<1 \%,<3 \%, 5 \%$, and $90 \%$, respectively. Plasma from a rat injected $30 \mathrm{~min}$ earlier with $0.5 \mathrm{ml}$ of saline showed the proportional LDH content for isoenzyme positions $1,2,3,4$, and 5 of $<2 \%,<1 \%$, $<1 \%$, $<1 \%$, and $96 \%$ of total $\mathrm{LDH}$, respectively. Thus, acute lung injury after the intravenous injection of CVF is associated with an LDH isoenzyme profile in plasma/serum that is consistent with origin from lung.

Lung injury, conjugated dienes, and $L D H$. After the intravenous injection of CVF, we examined extracts from plasma and lung for markers of injury and for evidence of products of lipid peroxidation. In Fig. 3 (upper graph), chloroform extracts from kidney, lung, and liver were obtained over a 1$h$ time period after injection of CVF. The dried residue, which was redissolved in heptane, was spectrophotometrically analyzed at $233 \mathrm{~nm}$ for an indication of conjugated dienes (14). For each time point at least six animals were used. No significant changes in content of conjugated dienes were noted in extracts from kidney, liver (Fig. 3, upper graph), and spleen (data not shown) over the 1-h period, although a significant increase in content of conjugated dienes was found in lungs at $30 \mathrm{~min}$, with nearly a doubling in the absorbance values compared to extracts from lungs of saline-injected rats. By $1 \mathrm{~h}$ the levels of conjugated dienes had returned to the normal range.

Changes in the lung content of conjugated dienes showed a close correlation with the appearance in plasma of the isoenzyme LDH-4 (Fig. 3, upper and middle graphs). Plasma activities of this enzyme reached a peak at $30 \mathrm{~min}$ and were approximately sixfold above the values for plasma obtained before the injection of CVF. Plasma LDH-4 levels were still significantly elevated after $60 \mathrm{~min}$ in CVF-injected rats. When lung injury was measured by the leakage of ${ }^{125} \mathrm{I}$-bovine serum albumin into the pulmonary parenchyma, there was a close correlation between increased lung vascular permeability and appearance of LDH-4 in the plasma (Fig. 3, middle graph). The pattern of changes in lung vascular permeability is similar to that in our earlier report (1).

Plasma content of conjugated dienes was also measured over a 2-h period of time after the injection of CVF. As shown in Fig. 3 (lower graph), by $10 \mathrm{~min}$ there were significant increases in plasma content of conjugated dienes, with steadily increasing levels that reached a peak at $30 \mathrm{~min}$, and with absorbance values that were nearly double those obtained in animals before injection of CVF. After the $30 \mathrm{~min}$ interval,

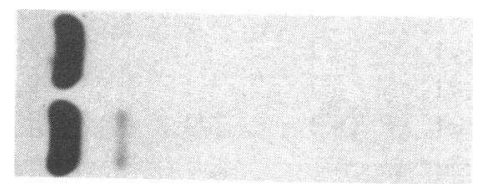

Figure 2. LDH isoenzyme patterns in plasma of rats injected 30 min earlier with saline or with cobra venom factor. The appearance of LDH isoenzyme in position 4 in the CVF-injected rat is suggestive of $\mathrm{LDH}$ origin from lung.
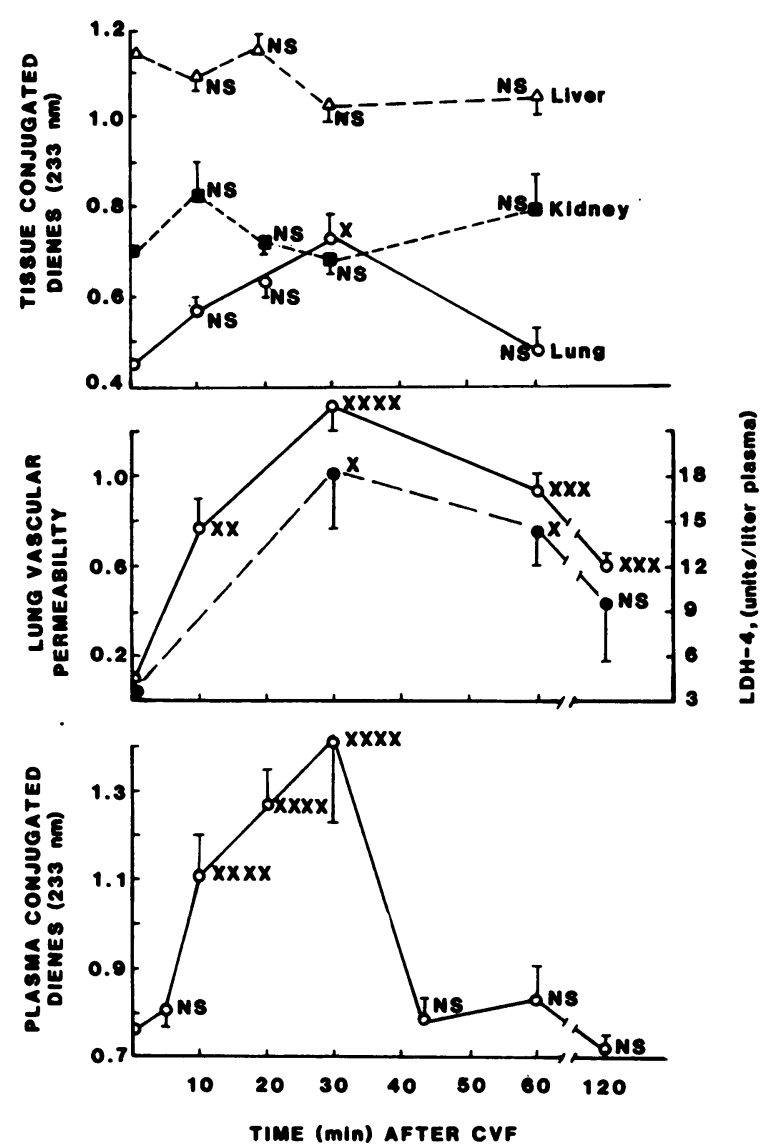

Figure 3. Lung injury and lipid peroxidation products in tissues and plasma of rats injected intravenously with CVF. In this and all succeeding figures, each point is the mean \pm SEM employing at least six different animals. The upper graph shows extractable conjugated dienes in the lungs, kidney, and liver. Only extracts from lung show a significant increase in conjugated dienes (at the $\mathbf{3 0}$ min interval). The middle graph shows the increasing lung vascular permeability ( $-0-$; measured by extravascular leakage of ${ }^{125} \mathrm{I}$-bovine serum albumin and expressed as the ratio of tissue reactivity to that in $1.0 \mathrm{ml}$ of blood), and appearance in plasma of LDH-4 isoenzyme (-.๑..). In the lower graph is the plasma content of conjugated dienes which reach a peak $30 \mathrm{~min}$ after the injection of CVF and then rapidly disappear. NS, not significant. $x, P<0.05 ; \times x, P<0.02 ; \times x \times, P$ $<0.005 ; \times x \times x, P<0.001$.

plasma levels of conjugated dienes in CVF-treated rats rapidly fell; by $\mathbf{4 5}$ min no significant increases in levels of conjugated dienes were found. These data indicate that lung injury after injection of CVF is closely associated with the appearance in plasma and in lung of increased levels of conjugated dienes as well as the appearance in plasma of LDH-4. Thus, lung injury (associated with lung vascular permeability changes and appearance of $\mathrm{LDH}-4$ ) correlates with the appearance in both lung and plasma of lipid peroxidation products (conjugated dienes) after systemic activation of complement.

In the oleic acid model of acute lung injury, four rats receiving the intravenous injection of oleic acid demonstrated extensive intrapulmonary hemorrhage and evidence of increased lung vascular permeability $(\bar{x} \pm \operatorname{SEM}=7.18 \pm 2.86)$ as compared to the values in four saline-injected rats $(0.14 \pm 0.01)$. When plasma levels of conjugated dienes were measured, the plasma 
Table II. Plasma Levels of Conjugated Dienes in CVF-treated Rats: Effects of Catalase, Neutrophil Depletion, Iron Chelators, and Hydroxyl Radical Scavengers

\begin{tabular}{|c|c|c|c|c|c|}
\hline \multirow[b]{2}{*}{ Treatment } & \multirow{2}{*}{$\begin{array}{l}\text { Number of } \\
\text { animals }\end{array}$} & \multicolumn{3}{|c|}{ Plasma levels of conjugated dienes } & \multirow{2}{*}{$\begin{array}{l}\text { Number of } \\
\text { neutrophils } \\
\text { in lung‡ }\end{array}$} \\
\hline & & $(\bar{x} \pm$ SEM) & Significance* & Changes & \\
\hline & $n$ & & $P$ values & $\%$ & \\
\hline Saline & 7 & $0.703 \pm 0.020$ & & & $0.35 \pm 0.15$ \\
\hline CVF & 6 & $1.571 \pm 0.115$ & & & $24.4 \pm 1.09$ \\
\hline CVF + PEG-catalase & 5 & $0.967 \pm 0.031$ & $<0.005$ & -70 & $24.4 \pm 0.87$ \\
\hline CVF + heated PEG-catalase & 3 & $1.508 \pm 0.123$ & NS & -7 & $23.7 \pm 0.83$ \\
\hline CVF + PEG-SOD & 3 & $1.447 \pm 0.103$ & NS & -14 & $22.6 \pm 1.02$ \\
\hline CVF + PMN depletion & 7 & $0.851 \pm 0.035$ & $<0.005$ & -83 & \\
\hline $\mathrm{CVF}+$ apolactoferrin & 3 & $0.911 \pm 0.010$ & $<0.010$ & -76 & $26.2 \pm 1.16$ \\
\hline CVF + iron-saturated lactoferrin & 4 & $1.329 \pm 0.061$ & NS & -28 & $25.7 \pm 1.04$ \\
\hline CVF + deferoxamine & 7 & $0.903 \pm 0.028$ & $<0.001$ & -77 & $22.0 \pm 0.82$ \\
\hline CVF + iron-saturated deferoxamine & 5 & $1.481 \pm 0.061$ & NS & -10 & $22.3 \pm 0.85$ \\
\hline CVF + DMSO & 4 & $0.987 \pm 0.058$ & $<0.001$ & -67 & $23.7 \pm 0.83$ \\
\hline CVF + DMTU & 5 & $0.946 \pm 0.068$ & $<0.005$ & -72 & $24.1 \pm 0.88$ \\
\hline Oleic acid & 4 & $0.770 \pm 0.044$ & NS§ & & \\
\hline
\end{tabular}

* Compared to the values of CVF-injected animals. NS, not significant. $¥$ Mean number of neutrophils in 40 different microscopic fields obtained from eight different lung sections. Values represent $\bar{x} \pm$ SEM numbers of neutrophils per microscopic field (at $\times 40$ ). $\S$ Compared to the value of saline-injected animals.

extracts from the oleic acid-injected rats were $0.770 \pm 0.044$, a mean value not significantly different from those obtained in saline-injected animals (Table II). These data suggest that the appearance of conjugated dienes is correlated with oxygen radical-mediated lung injury (after injection of CVF) but not with oxygen radical-independent lung injury (produced by oleic acid). The values for plasma levels of total LDH and LDH-4 in the oleic acid model were $1,217 \pm 174$ and $19.3 \pm 5.69$ U/liter, respectively (Table III). No detectable serum levels of LDH-1, 2, or 3 were found in these sera.

Comparison of ultraviolet absorption of plasma extracts from saline-injected and CVF-injected animals. Chloroform extracts (which were dried and redissolved in heptane) from the plasma of animals injected $30 \mathrm{~min}$ earlier either with 0.5 $\mathrm{ml}$ of saline or $0.5 \mathrm{ml}$ of CVF were compared for ultraviolet absorbance patterns. A representative pattern is shown in Fig. 4 , indicating a clear difference in the absorbance patterns of extracts from the two samples. In comparison to the chloroform extract of plasma from an animal before the injection of CVF, there was a sharp peak of increased absorbance in the 215235-nm range in the plasma extract of a CVF-injected animal. This extract also showed a broad increase in absorbance between 240 and $250 \mathrm{~nm}$ when compared to the plasma extract from a saline-treated rat. In vitro incubation of normal rat plasma and CVF at $37^{\circ} \mathrm{C}$ for $30 \mathrm{~min}$ did not result in any increases in ultraviolet absorbance in similarly processed extracts. These findings are consistent with the interpretation that in plasma of CVF-treated rats several different products, which partition into the chloroform layer, are present in increased amounts, including material with a maximal absorbance in the 230-nm region, suggestive of conjugated dienes.

Lipid peroxidation products in plasma and lung. To determine the pattern and the type of lipid peroxidation products present in CVF-injected animals, we analyzed both plasma and lung for evidence of conjugated dienes, hydroperoxides, malondialdehyde activity, and the presence of fluorescent

Table III. Plasma Levels of LDH, LDH-4, Hydroperoxides, and Fluorescent Products: Effects of Protective Interventions

\begin{tabular}{|c|c|c|c|c|c|c|c|c|}
\hline \multirow[b]{2}{*}{ Treatment } & \multicolumn{2}{|c|}{ Total LDH* } & \multicolumn{2}{|c|}{ LDH-4* } & \multicolumn{2}{|c|}{ Hydroperoxides* } & \multicolumn{2}{|c|}{ Fluorescent products* } \\
\hline & $n$ & U/liter & $n$ & U/liter & $n$ & $O D, 353 \mathrm{~nm}$ & $n$ & $O D, 430 \mathrm{~nm}$ \\
\hline Saline & 4 & $132 \pm 34.0$ & 4 & $<1.1$ & 3 & $0.032 \pm 0.008$ & 3 & $11.7 \pm 1.78$ \\
\hline CVF & 5 & $835 \pm 136$ & 3 & $18.8 \pm 0.94$ & 3 & $1.186 \pm 0.106$ & 3 & $72.2 \pm 7.43$ \\
\hline CVF + neutrophil depletion & 4 & $154 \pm 16.2$ & 4 & $3.4 \pm 0.61$ & 3 & $0.031 \pm 0.004$ & 3 & $36.5 \pm 1.02$ \\
\hline CVF + PEG-catalase & 3 & $345 \pm 124$ & 3 & $6.4 \pm 1.0$ & 3 & $0.019 \pm 0.005$ & 3 & $34.3 \pm 6.63$ \\
\hline $\mathrm{CVF}+\mathrm{DMSO}$ & 3 & $201 \pm 37.9$ & 3 & $3.9 \pm 0.42$ & 3 & $0.47 \pm 0.009$ & 3 & $14.4 \pm 3.13$ \\
\hline CVF + DMTU & 6 & $248 \pm 28.7$ & 5 & $5.27 \pm 0.84$ & & & & \\
\hline Oleic acid & 4 & $1,217 \pm 174$ & 4 & $19.3 \pm 5.69$ & & & & \\
\hline
\end{tabular}

* LDH and LDH-4 measurements were done on plasma samples obtained 30 min after injection of cobra venom factor; hydroperoxide assays were done on 15-min samples and assays for fluorescent products were done on 60 -min plasma samples. 


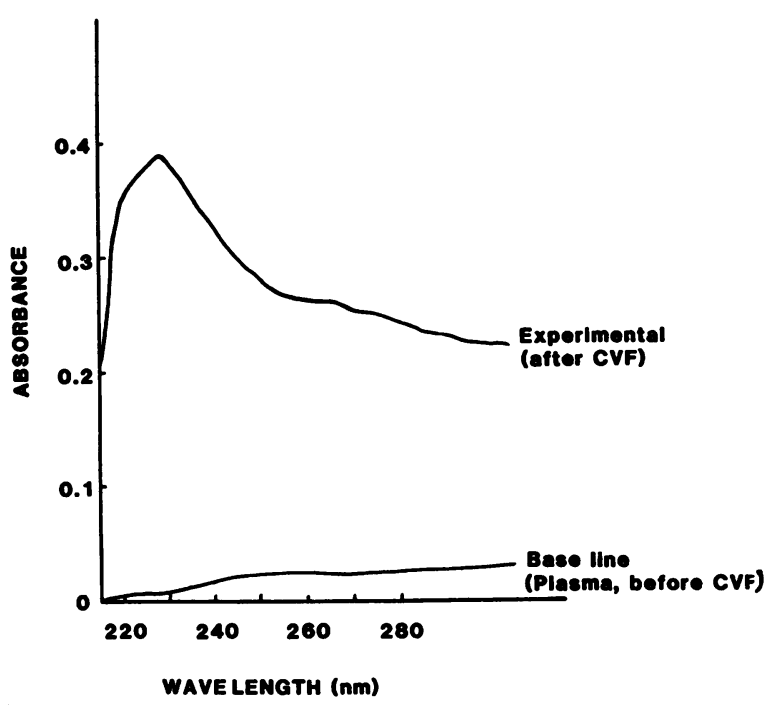

Figure 4. Ultraviolet absorbance patterns of chloroform extracts from plasma of a rat before and $30 \mathrm{~min}$ after the injection of CVF. The base line has been set with the extract of plasma from the salineinjected rat. The extract from the CVF-injected rat shows a broad elevation of ultraviolet absorbance with a peak around $230 \mathrm{~nm}$.

compounds characteristic of conjugated Schiff bases (16-18). As shown in Fig. 5 (upper graph), the plasma of rats injected with CVF showed large increases in levels of hydroperoxide, which reached a peak in $15 \mathrm{~min}$ and then rapidly diminished. Based upon comparisons with a reference curve established with cumene hydroperoxide, an absorbance of 1.0 in Fig. 5 (upper graph) can be extrapolated to a value of $75 \mathrm{nmol} / \mathrm{liter}$ of hydroperoxide. The levels of conjugated dienes, as expected, were maximal at $30 \mathrm{~min}$ and declined thereafter. When the same plasma samples were examined for the presence of fluorescent compounds that can be excited at $360 \mathrm{~nm}$ to emit maximally at $430 \mathrm{~nm}$, there were progressive increases in the plasma levels of fluorochromes, with a steady increment over the entire 60-min period (Fig. 5, lower graph). As was the case for hydroperoxides, these fluorochromes were present in the methanol-water extract of plasma but not in the chloroform extract (data not shown). When thiobarbituric acid-reactive material (malondialdehyde) was examined in plasma extracts over the hour-long interval, no increases in plasma levels of malondialdehyde in CVF-injected animals were noted (Fig. 5, lower graph). The failure to detect in plasma malondialdehyde activity is consistent with evidence that plasma malondialdehyde is an unreliable indicator in vivo of lipid peroxidation (reviewed in references 5,6 , and 18 ).

We also examined extracts from lungs of CVF-treated rats (Fig. 6). The products analyzed were similar to those described in Fig. 5. In accord with the data in Fig. 3, conjugated dienes could be found in chloroform extracts of rats injected with CVF 30 min earlier; the absorbance values of the chloroform extracts of lungs were approximately double those of controls (Fig. 6). In the case of fluorochrome products and malondialdehyde, both of which were measured in lung extracts obtained 60 min after injection of CVF, no significant increases in lung content of these materials were found. In addition, we were unable to detect increases in the concentration of hydroperoxides in lung extracts obtained $15 \mathrm{~min}$ after the injection

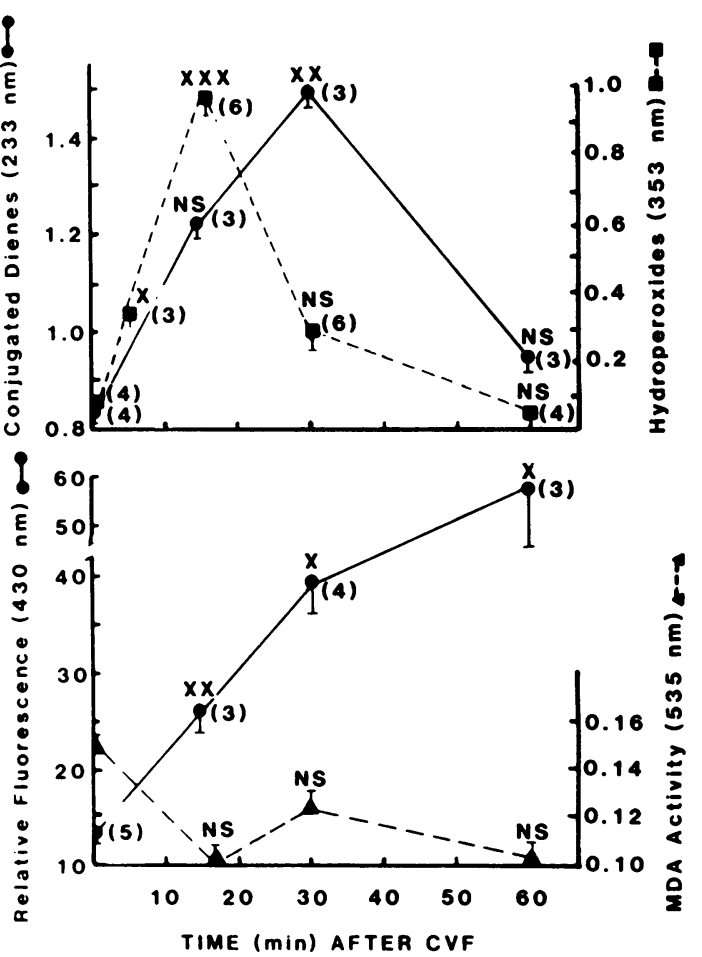

Figure 5. Products of lipid peroxidation in the plasma of rats over a 60 min time interval after injection of CVF. As expected, conjugated dienes reached a peak 30 min after injection of CVF whereas hydroperoxides peaked in the first $15 \mathrm{~min}$ (upper graph). No increase in malondialdehyde activity was demonstrable in the plasma over the hour-long period of time, but there was a progressive increase in the appearance of fluorescent products in the plasma of CVF-injected rats (lower graph). $\times, P<0.01 ; \times \times, P<0.005 ; \times \times \times, P<0.001$. NS, not significant.

of CVF (data not shown). A possible explanation for this apparent discrepancy is given in a later section.

Effects of protective interventions on plasma levels of con-

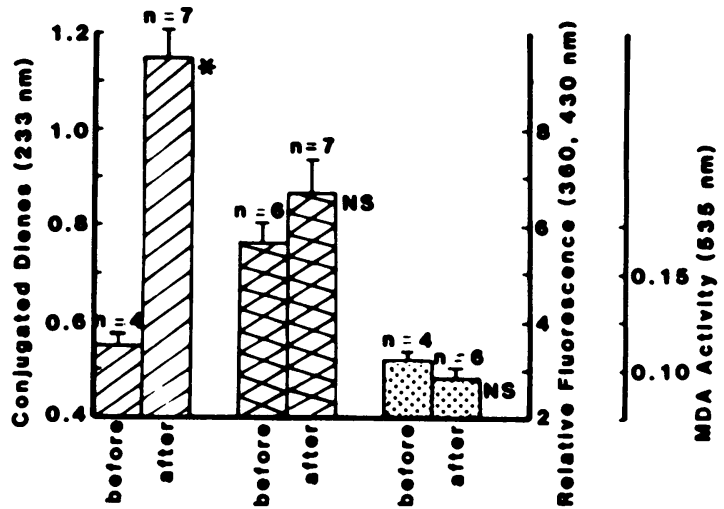

Figure 6. Products of lipid peroxidation in extracts of lungs after the intravenous injection of CVF. As expected, there was a sharp increase of conjugated dienes $(\square)$ in lungs 30 min after injection of CVF. There was no statistically significant increase in the levels of fluorescent products ( $\otimes$ ) or malondialdehyde (MDA) (\$) in lungs obtained at the 60 -min interval. The relative fluorescent values were obtained with an excitation wave length of $360 \mathrm{~nm}$ and an emission wave length of $430 \mathrm{~nm}$. NS, not significant. $*, P<0.005$. 
jugated dienes, LDH-4, hydroperoxides, and fluorescent products. Because the data described above indicate a good correlation between acute lung injury (defined as increases in vascular permeability) plasma levels of total $\mathrm{LDH}, \mathrm{LDH}-4$, conjugated dienes, hydroperoxides, and fluorescent products, a series of experiments was conducted that employed interventions that have been recently shown to be protective against acute lung injury after systemic complement activation $(1,2)$. The results of a large number of experiments are shown in Table II where changes in plasma content of conjugated dienes (employing the chloroform extraction method) are correlated with interventions known to protect against complementmediated lung injury. It has been established that these interventions do not interfere with activation of the complement system $(1,2)$.

Animals injected with CVF showed in their plasma a doubling in the absorbance values indicative of the presence of conjugated dienes when compared to animals injected with saline $(1.571 \pm 0.115$ vs. $0.703 \pm 0.020$, respectively). In PEGcatalase-injected rats, the rise in plasma content of conjugated dienes was greatly reduced (to $0.967 \pm 0.031$ ), a $70 \%$ fall. Heat inactivation of PEG-catalase virtually abolished its ability to prevent rise in plasma levels of conjugated dienes. As expected, PEG-SOD had a limited effect on the rise in plasma levels of conjugated dienes. Extensive reductions in the rise of conjugated dienes were found in neutrophil-depleted rats (value of $0.851 \pm 0.035$ ). A similar result was obtained in animals injected with apolactoferrin, which conferred $76 \%$ protection against the rise in plasma levels of conjugated dienes (Table II). In contrast, the use of iron-saturated lactoferrin resulted in a much greater rise in plasma content of conjugated dienes in CVF-injected animals, which is consistent with the known loss of protective effects against lung injury when iron is added to apolactoferrin (2). As would be expected, treatment of animals with deferoxamine, another chelator of iron, was nearly as effective in reducing the rise in plasma of conjugated dienes as were interventions involving neutrophil depletion or injection of catalase or apolactoferrin (Table II). Finally, the lungprotective interventions employing the $\mathrm{HO}^{\circ}$ scavengers, DMSO and DMTU, greatly attenuated the rise in levels of conjugated dienes. Without exception, these data show a remarkable correlation between the ability of a variety of interventions to protect against acute lung injury $(1,2)$ and, at the same time, to attenuate the anticipated increases in plasma levels of conjugated dienes.

Additional studies were undertaken to determine the relationship between the protective interventions described in Table II and effects on appearance in plasma of total LDH, LDH-4 isoenzyme, hydroperoxides, and fluorescent products. The data are summarized in Table III. LDH assays were carried out on plasma samples obtained $30 \mathrm{~min}$ after the intravenous injection of CVF in saline while samples for the hydroperoxide and the fluorochrome assays were obtained 15 min and $60 \mathrm{~min}$, respectively, after intravenous injection of saline or CVF, in accord with the times at which these products would be expected to reach peak levels in plasma of CVFinjected rats (see above).

The intravenous injection of saline provided the reference values for total $\mathrm{LDH}, \mathrm{LDH}-4$, hydroperoxides, and fluorescent products. In CVF-injected rats there was approximately a sixfold increase in total plasma content of LDH and a 17-fold increase in levels of $\mathrm{LDH}-4$. An almost 40-fold increase was found in plasma levels of hydroperoxides, and a nearly sevenfold increase in levels of fluorescent products. Under conditions of neutrophil depletion, both $\mathrm{LDH}$ as well as LDH-4 were reduced to the levels found in saline-injected rats whereas the hydroperoxide levels fell back to the base-line values (established in saline-injected rats) and fluorescent products showed an $82 \%$ reduction when compared to values in CVF-injected rats that were neutrophil-intact. Treatment of rats with PEGderivatized catalase had very similar effects. In animals pretreated with DMSO all four parameters (LDH, LDH-4, hydroperoxides, and fluorescent products) fell back toward the baseline values obtained in saline-injected rats. Finally, similar data were obtained in animals pretreated with DMTU, although only LDH and LDH-4 were evaluated. In general, it can be concluded that interventions protective against CVF-induced acute lung injury (as measured by increased vascular permeability changes) correlate with attenuations in serum levels of conjugated dienes, LDH, LDH-4, hydroperoxides, and fluorescent products.

Absence of evidence for CVF-induced injury in liver and kidney. The data presented above raise the question as to whether systemic complement activation may lead to acute injury of liver or kidney (or of vascular beds in other organs) and the extent to which $\mathrm{LDH}$ and conjugated dienes may be contributed to by these extrapulmonary sources. The data presented above indicate that conjugated dienes can be found in extracts of lung but not in extracts of liver, kidney, or spleen after intravenous injection of CVF. To address more directly the question of liver or renal injury resulting from intravenous injection of CVF, two separate studies were carried out. Urine was collected over an 18-h period from 11 rats injected intravenously with CVF (using conditions described above) or from eight rats similarly injected with $0.5 \mathrm{ml}$ of saline. The proteinuria values $(\bar{x} \pm \mathrm{SEM})$ in these two groups were $16.3 \pm 1.55 \mathrm{mg}$ and $16.3 \pm 0.85 \mathrm{mg}$, respectively. Thus, using proteinuria as a guide, acute renal damage could not be detected after intravenous injection of CVF. It should be pointed out that if CVF is injected directly into the renal artery, acute renal injury does ensue with proteinuria (19). This injury is neutrophil- and complement-dependent and is probably a reflection of efficient entrapment of activated neutrophils into glomerular capillaries.

The question of whether acute hepatic injury developed after intravenous injection of CVF was also addressed by measuring serum levels of aspartate aminotransferase, alanine aminotransferase, alkaline phosphatase, and leucine aminopeptidase. Values in saline-injected rats $(n=4)$ were $105 \pm 3.41$, $43 \pm 2.2,187 \pm 20.8$, and $25 \pm 5.6 \mathrm{U} /$ liter, respectively, whereas in CVF-injected rats values were $119 \pm 6.3,43 \pm 1.1,163 \pm 7.1$, and $23 \pm 8.7 \mathrm{U} /$ liter, respectively. These findings suggest that injection of CVF does not produce detectable evidence of acute liver injury. The failure to detect evidence of renal or hepatic injury under these conditions correlates with the lack of changes in levels of conjugated dienes in extracts of livers and kidneys of CVF-injected animals and suggests that these organs do not contribute to the rising plasma levels of dienes and LDH-4 after injection of CVF.

Morphometric analysis of lung tissue. To determine whether protective interventions affect the accumulation of neutrophils in lung interstitial capillaries, morphometry (as described above) 
was carried out on sections of lung tissues. The data are shown in Table II. In rats injected intravenously with saline, the mean number of neutrophils in lung interstitial capillaries present in $\mathbf{4 0}$ different microscopic fields was $0.35 \pm 0.15$ cells per field, whereas in CVF-injected rats this value rose dramatically to $24.4 \pm 1.09$ neutrophils per microscopic field. Protective interventions of PEG-catalase, PEG-SOD, apolactoferrin, deferoxamine, DMSO, and DMTU were correspondingly associated with values of $24.4 \pm 0.87,22.6 \pm 1.02,26.2 \pm 1.16$, $22.0 \pm 0.82,23.7 \pm 0.83$, and $24.1 \pm 0.88$, respectively. Whether or not apolactoferrin and deferoxamine were iron-saturated (removing their protective effects), the numbers of neutrophils in lung interstitial capillaries were not affected (Table II). There was no significant statistical difference in neutrophil numbers under conditions of any of the protective interventions as compared to the CVF control. These data indicate that the agents with protective effects against CVF-induced lung injury do not interfere with accumulation of neutrophils within lung interstitial capillaries.

Conjugated diene production in PMA-stimulated neutrophils. To address the question of whether activated neutrophils might contribute to the rise in plasma levels of conjugated dienes, rat neutrophils were stimulated with $200 \mathrm{ng}$ of PMA and samples examined over a 1-h interval for appearance of conjugated dienes. As the data in Table IV indicate, under the conditions employed, we were unable to detect appearance of conjugated dienes. Thus, it does not seem likely that conjugated dienes observed in the plasma of rats undergoing complement activation are the result of oxygen radical-mediated autotoxic changes in neutrophils.

\section{Discussion}

The data presented in this study suggest that, when systemic activation of complement occurs, the resulting acute lung injury is associated with the appearance in plasma and in lung of various products of lipid peroxidation. Several different products have been monitored, including hydroperoxides, conjugated dienes, malondialdehyde, and the fluorochromes that have features of Schiff bases. A possible scheme of events in lipid peroxidation is outlined in Fig. 7. The initial step of hydrogen abstraction resulting in the formation of a conjugated diene (which has a characteristic absorbance at $233 \mathrm{~nm}$ ) is

Table IV. Absence of Conjugated Diene Formation in PMA-stimulated Rat Neutrophils

\begin{tabular}{ll}
\hline $\begin{array}{l}\text { Material added to } \\
\text { neutrophils* }\end{array}$ & $\begin{array}{l}\text { Conjugated diene content } \\
(\bar{x} \pm \text { SEM })\end{array}$ \\
\hline & $O D, 233 \mathrm{~nm}$ \\
HBSS, 60 min & $0.195 \pm 0.040$ \\
PMA, 0 min & $0.152 \pm 0.027$ \\
PMA, 5 min & $0.156 \pm 0.039$ \\
PMA, 10 min & $0.168 \pm 0.005$ \\
PMA, 20 min & $0.140 \pm 0.020$ \\
PMA, 60 min & $0.192 \pm 0.009$
\end{tabular}

* $2 \times 10^{6}$ rat neutrophils obtained from a glycogen induced peritoneal exudate were incubated with Hanks' balanced salt solution (HBSS) or with $200 \mathrm{ng}$ of PMA in $1.0 \mathrm{ml}$. For each interval of time assays were done in triplicate.

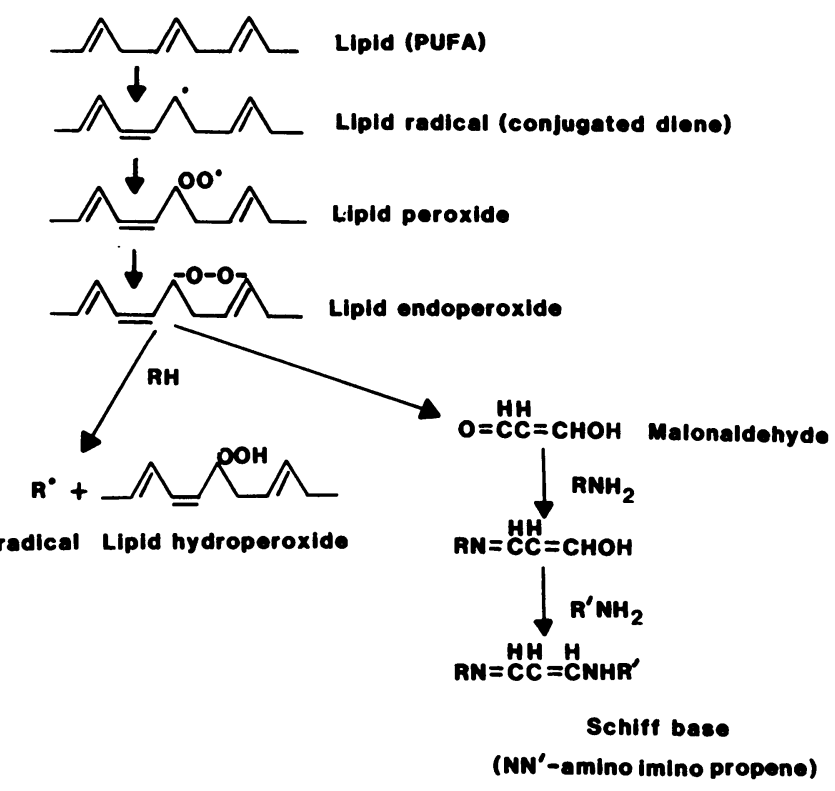

Figure 7. Postulated scheme of lipid peroxidation products from a polyunsaturated fatty acid (PUFA).

followed by the formation of a lipid peroxide and its conversion to a lipid endoperoxide. This endoperoxide can subsequently undergo reaction with a variety of compounds including hydrocarbons to form an alkyl radical and lipid hydroperoxide. Alternatively, the outcome may be the formation of malonaldehyde or malondialdehyde. Malonaldehyde is known to be highly reactive with $\epsilon$-amino groups of proteins resulting in either intramolecular bridging or intermolecular cross-linking, which results in the appearance of the characteristic fluorochromes (conjugated Schiff bases) with optimal excitation/ emission spectra at 360 and $430 \mathrm{~nm}$, respectively (3). Malonaldehyde and malondialdehyde also have the ability to react and bind with amino groups of phospholipids and aromatic amines $(16,18)$. When this occurs, and only when the aminoimino-propene compound is formed, can the fluorescent product be demonstrated (reviewed in references 5 and 6).

As the data in our report demonstrate, it has been possible to detect the presence of conjugated dienes in lung extracts of CVF-treated rats, although fluorochromic substances and hydroperoxides were not detectable. Analysis of plasma from the same animals revealed not only the appearance of conjugated dienes but also the presence of hydroperoxides and fluorochromic products. The inability to detect the latter two peroxidation products in extracts of lungs from CVF-treated rats could be due to the fact that, by transmission electron microscopy, pulmonary vascular endothelial cells are the chief targets of injury after systemic activation of the complement system (1). It would be expected that, if hydroperoxides and fluorochromic products are emanating from altered vascular endothelial cells, the materials could be released from surfaces of these damaged cells, to be carried away within the blood compartment. Thus, the inability to detect hydroperoxides and fluorochromic products in lung extracts of CVF-treated rats is not especially surprising. The striking relationship between interventions that protect against acute lung injury and parallel 
reductions in plasma levels of conjugated dienes, hydroperoxides, and fluorescent substances would suggest that there may be a direct relationship between lung injury and products of lipid peroxidation. Our studies do not prove that the appearance of products of peroxidation is relevant to the mechanism of lung vascular endothelial cell injury induced by oxygen radicals from activated neutrophils. Nevertheless, the ability to detect in a quantitative manner these products, which appear to bear a relationship to the intensity of acute lung injury, may be a useful method for monitoring conditions in which oxygen radical-mediated tissue damage is suspected.

Although acute lung injury is a consequence of intravenous injection of CVF, there is no convincing evidence of either acute renal or hepatic injury as indicated by data presented above. This is correlated with the findings of increased levels of conjugated dienes appearing in the lung but not in the liver and the kidney after injection of CVF. The data in Table IV suggest that the appearance of conjugated dienes may not result from autotoxic effects of oxygen radicals on neutrophils. Whereas vascular beds in areas other than those of liver and kidney may be damaged by CVF injection and thereby contribute to the conjugated diene and LDH content of plasma, the striking parallelism between lung injury (vascular permeability changes) and plasma or serum levels of $\mathrm{LDH}$ and conjugated dienes and the inverse quantitative relationship between all of these parameters and effects of protective interventions suggest that oxygen radical production and acute lung injury (and perhaps injury to other vascular beds) can be usefully monitored in the manner described in this paper.

There is good evidence in vitro that there is a predictable stoichiometric relationship between oxygen uptake by lipid and the appearance of conjugated dienes and production of fluorochromes (5). The latter are the result of the generation of malonaldehyde/malondialdehyde and the reactivity of this compound with amino groups as described above. The product with fluorescent properties is a $N, N^{\prime} 1$-amino-3-imino-propene compound, a conjugated Schiff base that is water- or lipidsoluble, depending on the origin of the reactive amino groups. In our studies the conjugated dienes partitioned into the organic (chloroform) phase whereas the hydroperoxides and fluorochrome products partitioned into the methanol-water phase, a pattern to be expected of hydrophobic and hydrophilic products, respectively. As has been pointed out in several studies, although malonaldehyde/malondialdehyde formation in vitro with peroxidized erythrocytes has been found, there has been a consistent inability to detect in vivo the presence in plasma of malonaldehyde/malondialdehyde $(5,6,18,20,21)$, probably because of its instability due to the presence of aldehyde oxidases within cells (22) as well as its reactivity with amino groups of proteins and phospholipids (see above).

Our data are consistent with others who have found fluorochrome products of lipid peroxidation in vitro under peroxidizing conditions utilizing amino-containing phospholipids and aromatic amino acids (3), enzymes such as ribonuclease A (20), and other proteins and nucleic acids (23). In experiments involving dietary manipulation (e.g., deficiency of selenium or vitamin $\mathrm{E}$ ) designed to increase susceptibility to oxidants, there has been evidence of loss of membraneassociated phospholipids (24), and fluorochromic compounds have been found in tissue extracts (reviewed in reference 5). Similar fluorescent compounds have also been found in erythrocytes of individuals treated with drugs known to have oxidant, hemolysis-inducing properties $(18,21,25)$ and in aging mice (26). Finally, the "aging pigment" lipofuscin found in human and in animal tissues has similar fluorescent properties to the products previously discussed (reviewed in reference 5).

As indicated above, there is as yet no formal proof that the hydroperoxides, conjugated dienes, and the fluorochrome products in plasma are derived from injured endothelial cells. It is possible that systemic complement activation leads to products of lipid peroxidation generated from neutrophils undergoing autotoxicity (although the data in Table IV suggest not) or from neutrophil-induced damage (by oxygen radicals) of erythrocytes. It can be reasonably assumed that any erythrocyte damage or lysis occurring in the CVF-injected rats is due not to oxygen radical-mediated damage of erythrocytes but is a direct effect of the membrane attack complex (C5b-9) generated in the course of complement activation. It has been recently shown in vitro that addition of CVF to guinea pig serum containing erythrocytes induced "bystander" lysis of the erythrocytes in the absence of leukocytes (27). In preliminary studies we have retrieved erythrocytes from rats injected 30 min earlier with CVF and have been unable to detect the presence of cell-associated conjugated dienes. Normal erythrocytes appear to be resistant to injury induced by oxygen radicals, a conclusion similar to that reached recently where human erythrocytes deficient in glucose-6-phosphate dehydrogenase or sickle cells are susceptible to oxygen radical-mediated injury (measured by appearance of malonaldehyde) while normal erythrocytes are resistant to injury (28). For all of these reasons it appears unlikely that the appearance of conjugated dienes in the plasma of CVF-injected rats is due to oxidant damage of erythrocytes. The quantitative relationship between lung injury (in the presence and absence of protective interventions) and tissue and plasma levels of conjugated dienes, hydroperoxides, and fluorescent products suggests that lung injury is associated with products of lipid peroxidation. It is possible that these products may be useful markers of oxygen radical-related injury in a variety of conditions such as acute respiratory distress syndrome and ischemic injury of myocardium and bowel where there is evidence that oxygen radicals may be involved in the full development of tissue injury $(29,30)$.

It should be noted that lipid peroxidation is not the exclusive mechanism for oxygen radical-induced damage in tissues. Superoxide anion, $\mathrm{O}_{2}^{\dot{2}}$, can enter into erythrocytes via anionic channels, resulting in the formation of methemoglobin and ultimate lysis of erythrocytes (31). Generation of oxygen radicals in lung may lead to secondary effects such as inactivation of $\alpha_{1}$-antiproteinase (32), the chief natural inhibitor of leukocytic neutral proteinases. As indicated above, oxygen radicals may also cause inactivation of critical cell-associated enzymes. Thus, no single biochemical event can exclusively explain the manner by which oxygen radicals bring about tissue injury.

Monitoring in vivo for the appearance of the volatile hydrocarbons ethane and pentane is reported to be a quantitative correlate of in vivo lipid peroxidation under conditions of susceptibility to oxidants. These products appear to result from the conversion of lipid peroxides to alkoxy radicals, which then undergo $\beta$-scission resulting in release of a volatile hydrocarbon. It has been possible to show the appearance of ethane or pentane in the breath of rats rendered deficient of 
selenium and vitamin E (33). Technically, these are difficult experiments in that alternative sources of the gases, especially from the gastrointestinal tract, require elaborate procedures to limit sampling to the expired breath.

The data presented in this report show the susceptibility of the lung to the damaging effects of the oxygen radical from neutrophils after systemic complement activation, using the ability to monitor plasma levels of the lung-related LDH isoenzyme profile. The conclusion that both lung injury as well as appearance of conjugated dienes are due to the role of $\mathrm{HO}^{\circ}$ is a tentative one, but is based on the strong $\mathrm{HO}^{\circ}$ scavenging effects of DMTU and DMSO $(34,35)$, and, perhaps most compellingly, the ability of deferoxamine but not ironsaturated deferoxamine to protect against lung injury (2) as well as markedly reducing the appearance of conjugated dienes in the plasma of animals undergoing systemic activation of complement. Why the lung should be the target for injury is intriguing. It could well be that the peripheral intravenous injection of CVF ensures by simple anatomic considerations that the rapidly produced leukoaggregates will be entrapped in the pulmonary interstitial capillary system, bringing about acute lung injury. The data presented above indicate the lack of evidence of acute renal or hepatic injury after intravenous injection of CVF. As indicated, it is possible to induce acute renal injury by the direct injection of CVF into the renal artery. The susceptibility of the lung to injury could be associated with susceptibility of pulmonary vascular endothelial cells to injury by oxygen radicals. It is known that, depending on their origin, vascular endothelial cells are quite distinct in certain metabolic and functional characteristics (reviewed in reference 36 ). In addition, the pulmonary vascular endothelium appears to be especially effective in the uptake of C5a (37). For these and other reasons it is possible that the pulmonary vascular lining is predisposed for injury produced by the numerous mechanisms discussed above.

In the CVF model of systemic complement activation and acute lung injury, whether or not vascular beds other than the lung contribute to the appearance of products of lipid peroxidation in the plasma cannot be determined. Nevertheless, increases in plasma levels of conjugated dienes, hydroperoxides, and fluorescent products appear to correlate with the extent of acute lung injury. It may be possible in human conditions, such as the adult respiratory distress syndrome, to use similar markers to detect and follow oxygen radical-mediated tissue injury.

\section{Acknowledgments}

This study was supported in part by grants GM-018832, GM-29507, HL-28442, and HL-31963 from the National Institutes of Health.

\section{References}

1. Till, G. O., K. J. Johnson, R. Kunkel, and P. A. Ward. 1982. Intravascular activation of complement and acute lung injury. Dependency on neutrophils and toxic oxygen metabolites. J. Clin. Invest. 69: 1126-1135.

2. Ward, P. A., G. O. Till, R. Kunkel, and C. Beauchamp. 1983. Evidence for role of hydroxyl radical in complement and neutrophildependent tissue injury. J. Clin. Invest. 72:789-801.

3. Dillard, C. J., and A. L. Tappel. 1973. Fluorescent products have reaction of peroxidizing polyunsaturated fatty acids with phosphatide ethanolamine and phenylalanine. Lipids. 8:183-189.
4. Aust, S. D., and B. A. Svingen. 1982. The role of iron in enzymatic lipid peroxidation. In Free Radicals in Biology, Vol. V. W. Pryor, editor. Academic Press, Inc., New York. 1-28.

5. Tappel, A. L. 1980. Measurement of and protection from in vivo lipid peroxidation. In Free Radicals in Biology, Vol. IV. W. Pryor, editor. Academic Press, Inc., New York. 1-47.

6. Chin, D., B. Lubin, and S. B. Shohet. 1982. Peroxidative reactions in red cell biology. In Free Radicals in Biology, Vol. V. W. Pryor, editor. Academic Press, Inc., New York. 115-160.

7. Dickey, B. F., R. S. Thrall, J. R. McCormick, and P. A. Ward. 1981. Oleic acid-induced lung injury in the rat. Failure of indomethacin treatment or complement depletion to ablate lung injury. Am. J. Pathol. 103:376-383.

8. Eiermann, G. J., B. F. Dickey, and R. S. Thrall. 1983. Polymorphonuclear leukocyte participation in acute oleic acid-induced lung injury. Am. Rev. Respir. Dis. 128:845-850.

9. Gay, R. J., R. B. McComb, and G. N. Bowers, Jr. 1968. Optimum reaction conditions for human lactate dehydrogenase isoenzymes as they affect total lactate dehydrogenase activity. Clin. Chem. 14:740-752.

10. Amador, E., M. F. Massod, and R. J. Franey. 1967. Reliability of glutamic-oxalacetic transaminase methods. Am. J. Clin. Pathol. 47: 419-428.

11. Henry, R. J., N. Chiamori, O. J. Golub, and S. Berkman. 1960. Revised spectrophotometric methods for the determination of glutamic-oxalacetic transaminase, glutamic-pyruvic transaminase, and lactic acid dehydrogenase. Am. J. Clin. Pathol. 34:381-398.

12. Nagel, W., F. Willig, and F. H. Schmidt. 1964. Über die Aminosäurearyl-amidase-(sog.Leucinaminopeptidase-) Aktivität im menschlichen Serum. Klin. Wochenschr. 42:447-449.

13. Wilkinson, J. H., J. H. Boutwell, and S. Winsten. 1969. Evaluation of a new system for the kinetic measurement of serum alkaline phosphatase. Clin. Chem. 15:487-495.

14. Buege, J. A., and S. D. Aust. 1978. Microsomal lipid peroxidation. Methods Enzymol. 52:302-310.

15. Ward, P. A., R. E. Duque, M. C. Sulavik, and K. J. Johnson. 1983. In vitro and in vivo stimulation of rat neutrophils and alveolar macrophages by immune complexes. Production of $\mathrm{O}_{2}^{\dot{\bar{\tau}}}$ and $\mathrm{H}_{2} \mathrm{O}_{2}$. Am. J. Pathol. 110:297-309.

16. Bidlack, W. R., and A. L. Tappel. 1973. Fluorescent products of phospholipids during lipid peroxidation. Lipids 8:203-207.

17. Gutteridge, J. M. C., and P. J. Kerry. 1982. Detection by fluorescence of peroxides and carbonyls in samples of arachidonic acid. Br. J. Pharmacol. 76:459-461.

18. Chio, K. S., and A. L. Tappel. 1971. Synthesis and characterization of the fluorescent products derived from malonaldehyde and amino acids. Biochemistry. 8:2821-2827.

19. Rehan, A., K. J. Johnson, R. C. Wiggins, and P. A. Ward. 1985. Oxygen free radical induced glomerular injury, following intravascular activation of complement. Fed Proc. 44:1546. (Abstr.).

20. Chio, K. S., and A. L. Tappel. 1971. Inactivation of ribonuclease and other enzymes by peroxidizing lipids and by malonaldehyde. Biochemistry. 8:2827-2832.

21. Goldstein, B. D., M. G. Rozen, and M. A. Amoruso. 1979. Relation of fluorescence in lipid-containing red cell membrane extracts to in vivo lipid peroxidation. J. Lab. Clin. Med. 93:687-694.

22. Horton, A. A., and L. Packer. 1970. Mitochondrial metabolism of aldehydes. Biochem. J. 116:16P.

23. Reiss, U., A. L. Tappel, and K. S. Chio. 1972. DNA-malonaldehyde reaction: formation of fluorescent products. Biochem. Biophys. Res. Commun. 48:921-926.

24. Jacob, H. S., and S. E. Lux IV. 1968. Degradation of membrane phospholipids and thiols in peroxide hemolysis: studies in vitamin $\mathrm{E}$ deficiency. Blood. 32:549-568.

25. Gladner, B. E., and M. E. Conrad. 1973. Hemolysis by 
diphenylsulfones: comparative effects of DDS and hydroxylamineDDS. J. Lab. Clin. Med. 81:267-272.

26. Tappel, A., B. Fletcher, and D. Deamer. 1973. Effects of antioxidants and nutrients on lipid peroxidation fluorescent products and aging parameters in the mouse. J. Gerontol. 28:415-424.

27. Vogel, C. W., and H. J. Muller-Eberhard. 1984. Cobra venom factor: improved method for purification and biochemical characterization. J. Immunol. Methods. 73:203-220.

28. Claster, S., D. T. Y. Chin, and B. Lubin. 1984. Neutrophils mediate lipid peroxidation in human red cells. Blood. 64:1079-1084.

29. Romson, J. L., B. G. Hook, S. L. Kunkel, G. D. Abrams, A. Schork, and B. R. Lucchessi. 1983. Reduction of the extent of ischemic myocardial injury by neutrophil depletion in the dog. Circulation. 67: 1016-1023.

30. McCord, J. M., and R. S. Roy. 1982. The pathophysiology of superoxide: roles in inflammation and ischemia. Physiol. Pharmacol. 60:1346-1352.

31. Weiss, S. J. 1980. The role of superoxide in the destruction of erythrocyte targets by human neutrophils. J. Biol. Chem. 255:99129917.
32. Cochrane, C. G., R. G. Spragg, and S. D. Revak. 1983. Studies on the pathogenesis of the adult respiratory distress syndrome. Evidence of oxidant activity in bronchoalveolar lavage fluid. J. Clin. Invest. 71: 754-761.

33, Dillard, C. J., E. E. Dumelin, and A. L. Tappel. 1977. Effect of dietary vitamin $\mathrm{E}$ on expiration of pentane and ethane by the rat. Lipids. 12:109-114.

34. Fox, R. B. 1984. Prevention of granulocyte-mediated oxidant lung injury in rats by a hydroxyl radical scavenger, dimethylthiourea. J. Clin. Invest. 74:1456-1464.

35. Parker, N. B., E. M. Berger, and J. E. Repine. 1984. Reaction with hydroxyl radical ('OH) causes disappearance of dimethylsulfoxide (DMSO). Clin. Res. 32:15a. (Abstr.)

36. Ryan, U. S., and J. W. Ryan. 1982. Vital and functional activities of endothelial cells. In Pathobiology of the Endothelial Cell. H. L. Nossel and H. J. Vogel, editors. Academic Press, Inc., New York. 455-469.

37. Webster, R. O., G. L. Larsen, and P. M. Henson. 1981. Tissue distribution of human C5a and C5a des arg complement fragments in normal and neutropenic rabbits. Am. Rev. Respir. Dis. 123:41. 